\title{
TRPA1 polymorphisms in chronic and complete spinal cord injury patients with neuropathic pain: a pilot study
}

\author{
Sonia Vidal Rodriguez ${ }^{1}{ }^{1} \cdot$ Inmaculada Castillo Aguilar $^{2} \cdot$ Luis Cuesta Villa $^{3} \cdot$ Francisco Serrano Saenz de Tejada $^{3}$
}

Received: 22 April 2017 / Revised: 25 July 2017 / Accepted: 25 August 2017

(C) International Spinal Cord Society 2017

\begin{abstract}
Study design Pilot study.
\end{abstract}

Objectives Single-nucleotide polymorphisms (SNPs) in TRPA1 gene are related to the etiology of chronic pain. The study is a pilot study with the primary objective of analyzing these SNPs in Spanish patients with chronic and complete spinal cord injury (SCI) and neuropathic pain (NPP).

Setting Asepeyo Hospital Department of Chronic and Complete SCI.

Methods Twelve patients with chronic and complete SCI and NPP, and 12 patients with chronic and complete SCI with no pain were reviewed. International Spinal Cord Injury Pain Classification (LANSS) and visual analog score (VAS) were chosen to classify pain syndrome. SNPs were identified by melting analysis after DNA amplification with real-time fluorescence PCR.

Results There were differences in rs11988795 variant: GG homozygous $(p=0.01)$ and G allele $(p=0.001)$ were more frequent in SCI patients with no pain. There were differences in rs 13255063 variant: TT homozygous were prevalent $(p=0.03)$ in patients with NPP.

Conclusions Until now this is the first study to show a description of TRPA1 SNPs in Spanish patients with chronic and complete SCI and NPP. These results suggest that GG genotype in rs11988795 variant and G allele could be protective factors against NPP. TT genotype in rs 13255063 variant could be a risk factor for NPP. Neuropathic pain after spinal cord injuries may have genetic contributions.

\section{Introduction}

According to data from the World Health Organization (WHO), nearly half a million people worldwide have been diagnosed with SCI. There are various causes of damage. Traffic accidents, accidents at work, and violent attacks are the most prevalent, based on available evidence across WHO regions [1]. There are many factors that lead to a loss

Sonia Vidal Rodriguez

svida168@gmail.com

1 Orthopaedic Research Department, ASEPEYO Hospital, Coslada 28820, Spain

2 Biomedicine Investigation Foundation Madrid 28015, Spain

3 Spinal Cord Injury Department, ASEPEYO Hospital, Coslada 28820, Spain of quality of life and of well-being after suffering SCI. Pain significantly reduces the adaptation to a new and different way of life [2].

Pain in SCI patients can be categorized into different groups [3]. Nociceptive pain arises from the activation of peripheral nerve endings (nociceptors) that respond to noxious stimulation. Nociceptive pain can be divided in two types. Musculoskeletal pain arises from nociceptors in muscles, tendons, ligaments, joints, or bones. If pain is identified in the thorax, abdomen, or pelvis and a specific pathology is present, it is classified as visceral pain. Many definitions of NPP have been used but the most accepted is a type of pain caused by damage to somatosensory system that alters the normal transmission of sensory signals. This pain is characterized by its persistence over time and high degree of heterogeneity. It is described as tingling, burning, squeezing, and pricking. Sharp, shooting, and painful hot/ cold are other characteristics of NPP. In turn NPP after SCI 
is divided into two subgroups [4]. At-level NPP has been described as pain, from damage to or disease of a nerve root or the spinal cord, that is experienced within three dermatomes below the neurologic level of injury, one dermatome above the level of injury, or both. Pain caused by injury in the cauda equine at-level NPP can be experienced up to six dermatomes below the level of injury.

Below-level SCI NPP is referred to pain more than three dermatomes below the level of injury, excluding pain related to damage in the cauda equine. SCI patients with complete and incomplete injuries can suffer below-level NPP. We consider that the field of SCI pain is intricately connected to both the SCI and pain research. Many factors have been studied to describe mechanisms of NPP [5]. The etiology remains controversial. It is related to the cellular damage caused by the liberation of neurotransmitters (glutamate and N-metil-D-aspartate) [6], and inflammatory cytokines [7]. Serotonin and noradrenergic metabolism disorders have been implicated [8] as well as molecular changes in sodium and calcium channels [9]. the most extensively studied receptor families in sensory biology are TRP channels. TRPA1 (TRP Ankyrin 1) and TRPV1 (TRP Vanilloid 1) are ion channels crucial for pain sensation [10]. They are predominantly expressed in peripheral sensory neurons, the trigeminal ganglia, dorsal root ganglia, sympathetic ganglia, and nodose ganglia. Several TRP channels have also been shown in the central nervous system and other tissues and cell types, such as fibroblasts, endotelial cells, and keratinocytes. TRPV1 and TRPA1 activation contributes to mechanical allodynia via spinal mechanisms and their interaction influences nociception [11]. In some studies co-expression of TRPA1 and TRPV1 was shown to inhibit TRPA1 agonist currents [12]. Even altering the interaction between these channels contributes to persistent pain [13]. Although the interaction between TRPA1 and TRPV1 is essential to understand the pathophysiology of pain, TRPA1 has been widely researched as the most important sensor of chemical damage and is involved in chronic and inflammatory pain. TRPA1 appears to play important roles in cold and mechanically evoked pain [14]. It is involved in the detection of cold hypersensitivity [15]. In humans, TRPA1 is not activated by heat [16].

TRPA1 channel plays an essential role in pain transduction and amplification. Its main function in nerve terminals is to be a chemosensor of nociception.TRPA1 is activated by various irritant chemicals and endogenous products of tissue injury. Acrolein, formalin, reactive oxygen species, nitrogen species, and carbony species are some of them [17]. Many studies have shown the importance of TRPA1 in mechanical nociception and primary hyperalgesia [18-20]. Also, a critical role in secondary (central) mechanical hyperalgesia has been shown [21]. Some studies have demonstrated that TRPA1 channels on spinal astrocytes contribute to central pain hypersensitivity [22]. A large number of experimental animal models have been developed to study the contribution of TRPA1 to pathophysiological pain. It is known that sleep deprivation aggravates pain and induces pain hypersensitivity. This process is partly mediated by spinal TRPA1 [23]. Sleep disturbances are frequent in SCI patients with NPP. In this line, it would be interesting to research the mechanisms mediated by TRPA1 in this process. Some studies provided evidence that blocking TRPA1 reduces pain hypersensitivity in peripheral neuropathy [24-26]. On the other hand, in spinal nerve-ligated animals, TRPA 1 mRNA is downregulated in the injured nerve and upregulated in the adjacent intact nerves and by this way, contribute to neuropahtic pain [27].

Research about genetic risk factors for chronic pain has experienced a boom over the last few years.

Many clinical studies on NPP after SCI report the frequency and characteristics of pain, but studies on pain based on genetic susceptibility are rare or non-existent.

In recent years three single-nucleotide polymorphisms (SNPs) have been described in TRPA1 associated with NPP in different populations. But none of these genetic changes have been studied in patients with SCI and NPP. SNPs are the most common type of genetic variation among people. Most of them have no effect on health or development but may help predict an individual's response to some drugs, susceptibility to environmental factors, and risk of developing particular diseases such as heart disease, diabetes, and cancer [28]. Association was found between short-duration cold pain sensitivity and rs11988795 $(2385+617 \mathrm{G}>\mathrm{A})$. This is an intronic SNPs, that means that it is situated between exons and is removed before translation of mRNA and does not function in coding for protein synthesis. In American females of European ancestry, A/A homozygote variants showed less pain tolerance to cold stimuli compared to G/G homozygotes [29].

In neuropathic pain patients, rs920829 (535 G > A; $710 \mathrm{G}>\mathrm{A}$, p.Glu179Lys) was associated with the presence of paradoxical heat sensation (PHS). Within the group of patients who suffered from PHS, heterozygous, and homozygous carriers of the TRPA1 $710 \mathrm{G}>\mathrm{A}$ variant were significantly underrepresented as compared to neuropathic pain patients without PHS [30]. This variant is an exonic SNP, that means it codes information for protein synthesis. In this case, the amino acid exchange from hydrophilic glutamate to hydrophobic lysine in codon 237 may result in alterations of expression and/or functionality of the protein, thus leading to a diminished sensitivity to noxious cold. In this way, some authors have studied these TRPA1 variants [31] in an in vitro expression system and observed that the variant Lys-179 TRPA1 failed to get activated by cold possibly due to the loss of ability to interact with 
other proteins or other TRPA1 monomers during oligomerization.

In other intronic variant: $r s 13255063(\mathrm{~A} / \mathrm{T}) \mathrm{A}$ is the minor allele and it has been described that homozygous have a higher pain threshold for electrical stimuli [32].

Genetic changes in TRPA1 are associated with neuropathic pain and the main objective of this study is to extrapolate this current evidence to complete and chronic SCI patients with this type of pain.

\section{Methods}

\section{Type of study}

Pilot study.

\section{Patients}

The population was selected from the Department of Complete and Chronic Spinal Cord Injuries and Rehabilitation, Asepeyo Hospital, Coslada, Madrid, Spain. Patients were enrolled from August 2016 to March 2017.

Each participant was required to sign an informed consent form, which had been approved by the Medical Ethics Committees of Asepeyo Hospital, Coslada, Madrid, Spain (affiliated with La Princesa Hospital, Madrid, Spain). A total of 24 chronic and complete SCI patients (21 males and 3 females) participated in this pilot study. Twelve SCI patients suffered NPP (cases group) and 12 SCI patients with no pain (control group).

American Spinal Injury Association (ASIA) Impairment Scale was the instrument selected to describe the extent of injury, and Spanish validation of the LANSS Scale (Leeds Assessment of Neuropathic Symptoms from the International Spinal Cord Injury Pain Classification) to classify neuropathic symptoms. Three clinicians carried out the assessments: the main author of this study (MD, $\mathrm{PhD}$ ) and two co-authors (MD). Visual analog score (VAS) was also used to measure pain. The localization of pain was also classified according to International Association for the Study of Pain criteria as NPP at the lesion level or below the lesion level.

LANSS score of $>12$ and VAS score of $>3$ were inclusion criteria in the cases group. Other inclusion criteria in the cases group were: (1) history of SCI $>5$ years (2) Neuropathic pain lasting $>6$ months. The treatments administered to the patients for NPP were also recorded. All patients with NPP were on ineffective first line treatment and they continued with symptoms of NPP. For this reason they were included in the cases group. After each patient was enrolled, a second line treatment was introduced to relieve pain.
SCI patients with LANSS score of 0 and VAS score of 0 were enrolled in the control group. Other inclusion criteria in the control group were: (1) history of SCI $>5$ years, (2) No pain lasting $>6$ months, and (3) any drug treatment.

The Spanish version of Modified Barthel Index was selected for measuring physical incapacity. The inability to follow commands or to give consent and the presence of pregnancy, chronic diseases like diabetes, cardiovascular diseases, oncology pathologies, chronic emotional distress, and smoking habit were exclusion criteria for both groups. SCI-related complications such as neurogenic bladder, neurogenic bowel, spasticity, decubitus ulcer, deep vein thrombosis, pulmonary embolism, and sexual problems were also exclusion criteria for both groups.

\section{Determination of genotypes}

Sampling was carried out with a pin prick to the finger using a sterile, single-use lancet, and the sample was deposited onto a card sampler. To extract total DNA from blood samples deposited on the sampling cards, a $5 \times 5-\mathrm{mm}$ fragment was clipped. Fragments were washed in $1 \mathrm{ml}$ of distilled $\mathrm{H}_{2} \mathrm{O}$ with orbital shaking for $15 \mathrm{~min}$. The samples were subjected to centrifugation for $3 \mathrm{~min}$ at $12,500 \times \mathrm{g}$. After discarding the supernatant, $1 \mathrm{ml}$ of distilled $\mathrm{H}_{2} \mathrm{O}$ was added, and washing and centrifugation were repeated. After removal of the supernatant, the card fragment was placed into another tube with $60 \mu \mathrm{l}$ of Tris-EDTA buffer solution; $\mathrm{pH}$ 7.5. and incubated at $50{ }^{\circ} \mathrm{C}$ for $15 \mathrm{~min}$. Subsequently, the card fragment was flattened at the bottom of the tube and was re-incubated at $97^{\circ} \mathrm{C}$ for $15 \mathrm{~min}$. Finally, the tubes were centrifuged for $4 \mathrm{~s}$ in a microcentrifuge, and $50 \mu \mathrm{l}$ of the supernatant was collected and placed into a new tube that was stored at $-20{ }^{\circ} \mathrm{C}$ until the determination of SNPs in TRPA1. SNPs were detected by melting analysis after DNA amplification with a real-time fluorescence PCR performed in a LightCycler 2.0 instrument (Roche Diagnostics, Mannheim, Germany) using the LightCycler FastStar DNA Master HybProbe kit (Roche Diagnostics) and fluorogenic probes (LightSNiPs, TIB MolBiol, Berlin, Germany), in accordance with the instructions supplied by the manufacturer.

\section{Statistical analysis}

The significance of this study was evaluated by the $\chi^{2}$ test. The association between the polymorphisms and neuropathic pain was estimated by the odds ratio (OR) and their $95 \%$ confidence intervals (CI). $p$-value was considered significant at $<0.05$. Differences related to allelic and genotyped frequencies were evaluated using Fisher's exact test. 
Table 1 Epidemiological and clinical characteristics

\begin{tabular}{llllll}
\hline Variable & $\begin{array}{l}\text { Total } n=24 n \\
(\%)\end{array}$ & $\begin{array}{l}\text { Cases }=12 n \\
(\%)\end{array}$ & $\begin{array}{l}\text { Controls =12n } \\
(\%)\end{array}$ & $p$-value & OR IC (95\%) \\
\hline Caucasians & $22(91.67)$ & $11(91.67)$ & $11(91.67)$ & NA & - \\
Africans & $2(8.33)$ & $1(8.33)$ & $1(8.33)$ & NA & - \\
Age (mean years) & 53.24 & 50.28 & 47.42 & 0.75 & $(-21.02-15.30)$ \\
Men & $21(87.5)$ & $11(91.67)$ & $10(83.33)$ & 0.5 & $1.10(0.81-1.49)$ \\
Women & $3(12.5)$ & $1(8.33)$ & $2(16.67)$ & 0.5 & $0.50(0.05-4.81)$ \\
History SCI (mean & 13.49 & 12.14 & 14.84 & 0.52 & $(-5.81-11.21)$ \\
years) & & $12(100)$ & $12(100)$ & NA & - \\
ASIA grade A & $24(100)$ & & & &
\end{tabular}

NA Not applicable, ASIA American Spinal Injury Association

Table 2 Clinical characteristics

\begin{tabular}{|c|c|c|c|c|c|c|}
\hline Subject $N^{\circ}$ & Level of sci & History sci (years) & Pain location & Treatment no relieved pain & Relieved pain & Barthel total points \\
\hline 1 & L1 & 14 & Below level & Amitriptyline + NSAIDs & +Relaxation training & 105 \\
\hline 2 & T9 & 15 & No pain & - & - & 105 \\
\hline 3 & $\mathrm{~T} 12$ & 11 & No pain & - & - & 97 \\
\hline 4 & $\mathrm{~T} 12$ & 13 & Below level & NSAIDs + relaxation training & + Lamotrigine & 105 \\
\hline 5 & $\mathrm{~L} 1$ & 12 & Below level & NSAIDs + GBP & +Relaxation training & 48 \\
\hline 6 & L1 & 10 & Below level & Lamotrigine + NSAIDs & +Relaxation training & 69 \\
\hline 7 & $\mathrm{C} 3$ & 9 & No pain & - & - & 0 \\
\hline 8 & $\mathrm{~T} 11$ & 11 & Below level & NSAIDs + relaxation training & + Lamotrigine & 62 \\
\hline 9 & $\mathrm{~T} 8$ & 12 & Below level & NSAIDs + GBP & + Relaxation training & 22 \\
\hline 10 & $\mathrm{C} 4$ & 7 & At level & Amitryptiline + NSAIDs + GBP & + Relaxation training & 0 \\
\hline 11 & $\mathrm{C} 7$ & 12 & At level & NSAIDs + GBP & +Relaxation training & 12 \\
\hline 12 & $\mathrm{C} 2$ & 13 & At level & NSAIDs + relaxation training & $+\mathrm{GBP}$ & 0 \\
\hline 13 & $\mathrm{~T} 10$ & 17 & No pain & Bapentine & - & 62 \\
\hline 14 & L1 & 16 & No pain & - & - & 105 \\
\hline 15 & $\mathrm{C} 3$ & 19 & At level & NSAIDs + Lamotrigine & +Relaxation training & 0 \\
\hline 16 & $\mathrm{~T} 10$ & 12 & Below level & Lamotrigine + NSAIDs & +Relaxation training & 62 \\
\hline 17 & L1 & 9 & No pain & - & - & 105 \\
\hline 18 & $\mathrm{C} 4$ & 6 & No pain & - & - & 0 \\
\hline 19 & $\mathrm{~T} 10$ & 14 & No pain & - & - & 105 \\
\hline 20 & $\mathrm{C} 3$ & 12 & No pain & - & - & 0 \\
\hline 21 & L1 & 19 & No pain & - & - & 105 \\
\hline 22 & $\mathrm{~T} 12$ & 13 & No pain & - & - & 62 \\
\hline 23 & $\mathrm{C} 2$ & 17 & At level & Lamotrigine + NSAIDs & +Relaxation training & 0 \\
\hline 24 & $\mathrm{~L} 1$ & 16 & No pain & - & - & 105 \\
\hline
\end{tabular}

\section{Results}

\section{Characteristics of subjects}

The characteristics of the overall population are shown in Table 1. Most of them were Caucasian and only two African SCI patients were identified.

In Table 2 clinical characteristics are shown. A complete and chronic SCI injury (ASIA score of A) was present in all patients. Most patients had below-level NPP (7/12:58.33\%) and only five patients had at-level NPP (5/12:41.67\%). Non-steroidal anti-inflammatory drugs (NSAIDs) were present in the treatment of all patients NPP. All patients required additional treatments to relieve the pain and relaxation training was the most frequent.

\section{Genotypes and alleles in controls and cases}

The genetic and allelic frequencies for the different SNPs are shown in Tables 3 and 4. 
Table 3 Genetic variables

\begin{tabular}{llllll}
\hline TRPA1 & Total $N=24 n(\%)$ & Cases $N=12 n(\%)$ & Controls $N=12 n(\%)$ & $p$-value & OR (IC-95\%) \\
\hline$r s 920829$ & & & & \\
GG & $17(70.83)$ & $7(58.33)$ & $10(83.33)$ & 0.2 & $0.70(0.41-1.20)$ \\
AG & $4(16.67)$ & $3(25)$ & $1(8.33)$ & 0.3 & $3.0(0.36-24.9)$ \\
AA & $3(12.5)$ & $2(16.67)$ & $1(8.33)$ & 0.5 & $2.0(0.21-19.23)$ \\
$r s 11988795$ & & & & \\
GG & $13(54.17)$ & $1(8.33)$ & $12(100)$ & 0.01 & $0.08(0.013-0.544)$ \\
AG & $7(29.17)$ & $7(58.33)$ & $0(0)$ & 0.05 & $15(0.95-236.4)$ \\
AA & $4(16.67)$ & $4(33.33)$ & $0(0)$ & 0.13 & $9(0.53-150.8)$ \\
$r s 13255063$ & & & & \\
TT & $14(58.33)$ & $10(83.33)$ & $4(33.33)$ & 0.03 & $2.2(1.9-5.8)$ \\
AT & $5(20.83)$ & $2(16.67)$ & $3(25)$ & 0.6 & $0.7(0.13-3.30)$ \\
AA & $5(20.83)$ & 0 & $5(41.67)$ & 0.09 & $0.1(0.05-1.48)$ \\
\hline
\end{tabular}

\section{rs920829}

There were no significant differences between both groups in genotypes neither in allelic frequencies.

\section{rs11988795}

There were differences in homozygous GG in SCI patients with no pain, $p=0.01$, compared with the cases group. $\mathrm{G}$ allele was more frequent in the control group $(p=0.001)$.

\section{rs13255063}

There were differences in homozygous TT in SCI patients with NPP, $p=0.03$, compared with the control group. There were no differences related to allelic frequencies.

\section{Discussion}

Neuropathic pain is a common complication after injuries in the spinal cord and causes extremely adverse effects on well-being and lives of SCI patients. It is complex and usually requires a combination of treatment approaches to manage. We subscribe to the definition of chronic pain proposed by the International Association for the Study of Pain. Chronic pain is a type of pain without biological value that persisted beyond the normal healing time. This may be $<1$ month to more than 6 , with 3 months being considered a convenient separation line between acute and chronic pain [33]. Our inclusion criteria are in line with these definitions. The Barthel Index was published by Barthel and Mahoney in 1965, after 10 years of using it to measure functional disability in patients with stroke and musculoskeletal pathologies [34, 35]. In Asepeyo Rehabilitation Department we use the Spanish version [36]. The modified Barthel Index has high psychometric qualities and, in our opinion, is one of the best scales for assessing activities of daily living. It has a high degree of accuracy and reproducibility. The LANSS Pain Scale allows us to distinguish patients with neuropathic pain from those with nociceptive pain [37]. We have used the Spanish version [38] and three clinicians carried out the assessments. Some studies have reported NPP to be more common in patients with incomplete SCI [39]. This study sample comprises only A ASIA patients, with complete and chronic SCI because all of them were enrolled from the Asepeyo Hospital Department of Complete and Chronic Spinal Cord Injuries. This must be taken into consideration in future studies with other ASIA classification patients. We have not found differences in demographic features between the two groups. Gender and ethnicity are important variables in research for a better knowledge of genetic and environmental interactions as determinants of disease. In this study 21 males were identified and only 3 females. Most of them were Caucasian of Spanish origin This study was carried out from August 2016 to March 2017 and the results are only a description of this period of time. During this time, only two African SCI patients were attended in the hospital. One of them suffered NPP and the other no pain.

We are aware that the most important limitation of the current study is the small sample size and that the results should be interpreted with caution. But we are only presenting a pilot study and suggesting a future study with higher sample size and patients matched by age, gender, and ethnic group.

The pathophysiological mechanism involved in the development of NPP is not well understood. TRP family has been described as integral membrane proteins that function as ion channels and make a significant contribution to transduction and sensitization in primary afferent somatosensory neurons [40]. When TPR channels are activated, a depolarization of the cellular membrane occurs and this leads the flow of ions, such as potassium, calcium and 
Table 4 Allelic frequencies

\begin{tabular}{llllll}
\hline TRPA1 & Allele frequency & Cases $N=12(\%)$ & Controls $N=12(\%)$ & Mantel-Haenszel $\chi^{2}$ & $p$-value \\
\hline$r s 920829$ & $\mathrm{G}$ & $71 \%$ & $88 \%$ & 1.02 & 0.31 \\
& $\mathrm{~A}$ & $29 \%$ & $12 \%$ & & \\
rs 11988795 & $\mathrm{G}$ & $38 \%$ & $100 \%$ & 10.33 & 0.001 \\
& $\mathrm{~A}$ & $62 \%$ & $0 \%$ & & 0.7 \\
$r s 13255063$ & $\mathrm{~T}$ & $100 \%$ & $46 \%$ & 0.15 & \\
& $\mathrm{~A}$ & $0 \%$ & $54 \%$ & & \\
\hline
\end{tabular}

sodium. The final result is a nerve impulse and physiological sensation or perception. The discovery of TRP channels has revolutionized the research of many sensory processes. Mutations in several TRP genes have been implicated in diverse pathological states, including neurodegenerative disorders, skeletal dysplasia, kidney disorders, and chronic pain [41, 42].

In this pilot study we selected TRPA1 gene based on representation in literature and level of evidence in previous studies. It is a gene frequently investigated in genetic research on pain, but this is the first description of TRPA1 polymorphisms in Spanish Complete and Chronic SCI patients who suffer neuropathic pain. In vitro and in vivo animal studies have shown the role of TRP channels in the generation of NPP [43]. TRPA1 has been proposed to contribute to a variety of sensory processes, including thermal, nociception, mechanosensation, and inflammatory hyperalgesia [24]. Additionally, TRPA1 is expressed in neurons in regions where the function in thermosensation is known, such as the dorsal root or trigeminal ganglia. The importance lies in the fact that thermal allodynia (hot/cold) is a sensation commonly present in neuropathic pain. A SNP has been described: rs11988795 (G > A) that enhances sensitivity toward nociceptive cold stimuli [29]. Carriers of the minor A allele had a shorter withdrawal latency to noxious cold and in another small subgroup, this allele was associated with sensitivity to heat pain. In our results, there were no differences in the minor allele, A, but differences were shown in the GG genotype. Homozygous patients were more frequent in SCI patients with no pain. Also, G allele was prevalent in patients with no pain. These results lead us to think that GG genotype and $G$ allele protect against NPP in SCI patients. This means that these genetic variations are more frequent in SCI patients with no pain. So, the susceptibility to suffering NPP would be lower compared with patients with different genotypes and alleles. Based on our results, gene tests on specific SNPs might serve as predictors of pain qualities in the future. Another point to which we wish to draw attention is the interaction between TRP channels, especially TRPA1 and TRPV1. Both molecular properties are important. It has been clearly demonstrated that TRPA1 can oligermerize with TRPV1. The heteromerization can affect the calcium signaling pathways and modulates channel voltage relationships allowing muscle nociception and mechanical hyperalgesia [44]. Future studies including genetic changes in TRPA1 and TRPV1, with a higher sample size, could be useful to analyze haplotypes, associated with NPP in SCI patients. Somatosensory abnormalities that are present in patients who suffer NPP without SCI are mediated by the TRPA1 variant $r s 920829$ [30]. It has been described that patients who presented paradoxical heat in NPP showed a lower frequency of GA and AA genotypes compared with patients with no heat sensation. In our study there were no differences in this polymorphism. It has been described that the rs13255063 influences pain sensitivity after electrical stimuli [32]. There were more homozygous TT patients in the cases group. These results suggest that TT genotype could be an additional risk factor for developing neuropathic pain after spinal cord injuries. Gabapentin, pregabalin, and amitriptyline have proved effective in treating SCI patients with NPP. Gabapentin relieves at-level and below-level pain in paraplegics with complete SCI [45], although some authors disagree over these results [46]. In SCI with complete and incomplete injury treated with pregabalin, less pain and a better control of disturbing sleep have been demonstrated [47]. Amitriptyline has been studied in SCI patients with mixed neuropathic and nociceptive pain [48]. Based on current evidence, gabapentin, amitriptyline, and pregabalin have been considered the first choice in the treatment of SCI patients with NPP. Although a recent systematic review shows that anticonvulsants are the most studied and supported treatment for NPP in SCI patients [49]. Our results are in line with this evidence. All patients with NPP were on ineffective first line treatment and they continued with symptoms of NPP. For this reason they were included in the cases group. After each patient was enrolled, a second line treatment was introduced to relieve pain. Relaxation training was the most frequent and effective. Patients with NPP were trained in potentially useful therapies for dealing with pain, such as cognitive behavior therapy, biofeedback, relaxation strategies, and group counseling.

The time has come to consider TRPA1 for pain relief. A pre-clinical study proposes that TRPA1 agonism could contribute to prolonging the analgesic action of 
acetaminophen [50]. Authors suggested that the reactive metabolite of acetaminophen acts in vivo as a TRPA1 agonist and by such action attenuates pain through desensitizing TRPA1-expressing sensory neurons. This discovery appears exciting but must be taken cautiously because TRPA1 is a calcium-permeable nonselective cation channel and long-term sustained TRPA1 activation and possible pore dilation evoked by TRPA1 agonists can lead to excitotoxicity [51]. Moreover, TRPA1 antagonists have been designed and identified as potential therapeutic drugs [52]. Inhibitors of TRP channels hold the potential for the control of pain, asthma, and neurodegenerative diseases, which are associated with increased activities of TRP channels. In animal models, pharmacological inhibitors of TRPA1 have been shown to be effective in attenuating mechanical hyperalgesia associated with neuropathic pain conditions [53].

Until now, there have been no studies considering genetic risk factors in chronic and complete SCI patients with NPP. TRPA1 is an interesting pharmacological target due to its role in sensory nerve activation, pain and inflammation. The prospect of personalized medicine is a step towards promising pain management strategies in SCI patients with NPP and our preliminary results contribute to opening the door in this direction.

\begin{abstract}
Acknowledgements Spanish Abertis Foundation: This study was funded by Abertis Foundation of Spain. Abertis is the international market leader in the management of toll roads, managing over $8600 \mathrm{~km}$ of high capacity and quality roads around the world. Its ongoing internationalization process has led Abertis to be present in 14 countries in Europe, the America, and Asia, and this has enabled it to diversify its geographic risk and better adapt to global business cycles. Abertis is the first national toll road operator in countries such Spain and Chile, and has a highlighted presence in France, Brazil, Italy, and Puerto Rico. The company also has a stake in more than $700 \mathrm{~km}$ through different concessionaires in United Kingdom, Argentina, and Colombia. Over $70 \%$ of the Group's revenue is generated outside Spain. Especially important in this respect are France, Brazil, and Chile. Abertis is listed on the Spanish Stock Exchange and is on the IBEX 35 and the international FTS Eurofirst 300 and Standard \& Poor' Europe 350 indexes. The Abertis Foundation was established in 1999 as a non-profit organization, with the objective of responding to the impact that the Abertis Group's economic activity has in the different territories and countries where the Group is present. The Foundation has always prioritized actions related to road safety, the environment, and social action, in line with the Abertis Corporate Social Responsibility Strategic Plan.
\end{abstract}

Author contributions S.V.V.R. is first author and the principal researcher. All authors have read and approved the final submitted manuscript.

\section{Compliance with ethical standards}

Conflict of interest The authors declare that they have no competing interests.

\section{References}

1. Singh A, Tetreault L, Kalsi-Ryan S, Nouri A, Fehlings MG. Global prevalence and incidence of traumatic spinal cord injury. Clin Epidemiol. 2014;6:309-31.

2. Saadat S, Javadi M, Divshali BS, Tavakoli AH, Ghodsi SM, Montazeri A, et al. Health-related quality of life among individuals with long-standing spinal cord injury: a comparative study of veterans and non-veterans. BMC Public Health. 2010;10:6.

3. Esquenazi A, Talaty M, Packel A, Saulino M. Spinal cord injury pain. Phys Med Rehabil Clin N Am. 2014;25:397-410.

4. Jang JY, Lee SH, Kim MY, Ryu JS. Characteristics of neuropathic pain in patients with spinal cord injury. Ann Rehabil Med. 2014:38:327-34.

5. Cohen SP, Mao J. Neuropathic pain: mechanisms and their clinical implications. BMJ. 2014;348:f7656.

6. Liu XJ, Salter MW. Glutamate receptor phosphorylation and trafficking in pain plasticity in spinal cord dorsal horn. Eur J Neurosci. 2010;32:278-89.

7. Ji R-R, Xu Z-Z, Gao Y-J. Emerging targets in neuroinflammationdriven chronic pain. Nat Rev Drug Discov. 2014;13:533-48.

8. Ferjan I, Lipnik-Štangelj M. Chronic pain treatment: the influence of tricyclic antidepressants on serotonin release and uptake in mast cells. Mediators Inflamm. 2013;2013:340473.

9. Mickle AD, Shepherd AJ, Mohapatra DP. Nociceptive TRP channels: sensory detectors and transducers in multiple pain pathologies. Pharmaceuticals. 2016;9:72.

10. Marwaha L, Bansal Y, Singh R, Saroj P, Bhandari R, Kuhad A. TRP channels: potential drug target for neuropathic pain. Inflammopharmacology. 2016;24:305-17.

11. Akopian AN. Regulation of nociceptive transmission at the periphery via TRPA1-TRPV1 interactions. Curr Pharm Biotechnol. 2011;12:89-94.

12. Salas MM, Hargreaves KM, Akopian AN. TRPA1-mediated responses in trigeminal sensory neurons: interaction between TRPA1 and TRPV1. Eur J Neurosci. 2009;29:1568-78.

13. Weng HJ, Patel KN, Jeske NA, Bierbower SM, Zou W, Tiwari V, et al. Tmem100 is a Regulator of TRPA1-TRPV1 complex and contributes to persistent pain. Neuron. 2015;85:833-46.

14. Bautista DM, Pellegrino M, Tsunozaki M. TRPA1: a gatekeeper for inflammation. Annu Rev Physiol. 2013;75:181-200.

15. Del Camino D, Murphy S, Heiry M, Barrett LB, Earley TJ, Cook CA, et al. TRPA1 contributes to cold hypersensitivity. J Neurosci. 2010;30:15165-74.

16. Cordero-Morales JF, Gracheva EO, Julius D. Cytoplasmic ankyrin repeats of transient receptor potential A1 (TRPA1) dictate sensitivity to thermal and chemical stimuli. Proc Natl Acad Sci USA. 2011;108:E1184-E1191.

17. Andersson DA, Gentry C, Moss S, Bevan S. Transient receptor potential A1 is a sensory receptor for multiple products of oxidative stress. J Neurosci. 2008;28:2485-94.

18. Kwan KY, Glazer JM, Corey DP, Rice FL, Stucky CL. TRPA1 modulates mechanotransduction in cutaneous sensory neurons. J Neurosci. 2009;29:480.

19. Kerstein PC, Del Camino D, Moran MM, Stucky CL. Pharmacological blockade of TRPA1 inhibits mechanical firing in nociceptors. Mol Pain. 2009;5:19.

20. Treede RD, Meyer RA, Raja SN, Campbell JN. Peripheral and central mechanisms of cutaneous hyperalgesia. Prog Neurobiol. 1992;38:397-421.

21. Torebjörk HE, Lundberg LE, LaMotte RH. Central changes in processing of mechanoreceptive input in capsaicin-induced secondary hyperalgesia in humans. J Physiol. 1992;448: $765-80$. 
22. Wei H, Koivisto A, Saarnilehto M, Chapman H, Kuokkanen K, Hao B, et al. Spinal transient receptor potential ankyrin 1 channel contributes to central pain hypersensitivity in various pathophysiological conditions in the rat. Pain. 2011;152:582-91.

23. Lautenbacher S, Kundermann B, Krieg JC. Sleep deprivation and pain perception. Sleep Med Rev. 2006;10:357-69.

24. Obata K, Katsura H, Mizushima T, Yamanaka H, Kobayashi K, Dai $\mathrm{Y}$, et al. TRPA1 induced in sensory neurons contributes to cold hyperalgesia after inflammation and nerve injury. J Clin Invest. 2005;115:2393-401.

25. Katsura H, Obata K, Mizushima T, Yamanaka H, Kobayashi K, Dai Y, et al. Antisense knock down of TRPA1, but not TRPM8, alleviates cold hyperalgesia after spinal nerve ligation in rats. Exp Neurol. 2006;200:112-23.

26. Wei H, Viisanen H, Amorim D, Koivisto A, Pertovaara A. Dissociated modulation of conditioned place-preference and mechanical hypersensitivity by a TRPA1 channel antagonist in peripheral neuropathy. Pharmacol Biochem Behav. 2013;104:90-96.

27. Ringkamp M, Meyer RA. Injured versus uninjured afferents: who is to blame for neuropathic pain? Anesthesiology. 2005;103:221-3.

28. Murata M. Clinical significance and strategies to analyze genetic polymorphisms. Rinsho Byori. 2001;49:153-6.

29. Kim H, Mittal DP, Iadarola MJ, Dionne RA. Genetic predictors for acute experimental cold and heat pain sensitivity in humans. J Med Genet. 2006; 43(8):e40.

30. Binder A, May D, Baron R, Maier C, Tölle TR, Treede RD, et al. Transient receptor potential channel polymorphisms are associated with the somatosensory function in neuropathic pain patients. PLoS ONE. 2011;6:e17387.

31. May D, Baastrup J, Nientit MR, Binder A, Schünke M, Baron R, et al. Differential expression and functionality of TRPA1 protein genetic variants in conditions of thermal stimulation. J Biol Chem. 2012;287:27087-94.

32. Doehring A, Küsener N, Flühr K, Neddermeyer TJ, Schneider G, Lötsch J. Effect sizes in experimental pain produced by gender, genetic variants and sensitization procedures. PLoS ONE. 2011;6: e17724.

33. ASP Subcommitee on Taxonomy. Classification of chronic pain. Description of chronic pain syndromes and definition of pain terms. Pain Suppl. 1986;3:S1-226.

34. Mahoney FI, Barthel DW. Functional evaluation: The Barthel Index. Md State Med J. 1965;14:61-5.

35. Granger CV, Albrecht GL, Hamilton BB. Outcome of comprehensive medical rehabilitation: Measurement by PULSES profile and the Barthel Index. Arch Phys Med Rehabil. 1979;60:145-54.

36. Baztan JJ, Pérez del Molino J, Alarcón T, Marzanbeitia J. Indice de Barthel: instrumento válido para la valoración funcional de pacientes con enfermedad cerebrovascular. Rev Esp Geriatr Gerontol. 1993;28:32-40.

37. Bennett MI, Smith BH, Torrance N, Potter J. The S-LANSS score for identifying pain of predominantly neuropathic origin: validation for use in clinical and postal research. J Pain. 2005;6:149-58.
38. Pérez C, Gálvez R, Insausti J, Bennett M, Ruiz M, Rejas J, et al. Linguistic adaptation and Spanish validation of the LANSS (leeds assessment of neuropathic symptoms and signs) scale for the diagnosis of neuropathic pain. Med Clin. 2006;127:485-91.

39. McKinley W, Santos K, Meade M, Brooke K. Incidence and outcomes of spinal cord injury clinical syndromes. J Spinal Cord Med. 2007;30:215-24.

40. Tominaga M. Nociception and TRP channels. Handb Exp Pharmacol. 2007;179:489-505.

41. Vennekens R. Emerging concepts for the role of TRP channels in the cardiovascular system. J Physiol. 2011;589:1527-34.

42. Everett KV. Transient receptor potential genes and human inherited disease. Adv Exp Med Biol. 2011;704:1011-32.

43. Jardín I, López JJ, Diez R, Sánchez-Collado J, Cantonero C, Albarrán L, et al. TRPs in pain sensation. Front Physiol. 2017;8:392.

44. Staruschenko A, Jeske NA, Akopian AN. Contribution of TRPV1- TRPA1 interaction to the single channel properties of the TRPA1 channel. J Biol Chem. 2010;285:15167-77.

45. Levendoglu F, Ogün CO, Ozerbil O, Ogün TC, Ugurlu H. Gabapentin is a first line drug for the treatment of neuropathic pain in spinal cord injury. Spine. 2004;29:743-51.

46. Rintala DH, Holmes SA, Courtade D, Fiess RN, Tastard LV, Loubser PG. Comparison of the effectiveness of amitriptyline and gabapentin on chronic neuropathic pain in persons with spinal cord injury. Arch Phys Med Rehabil. 2007 ;88:1547-60.

47. Siddall PJ, Cousins MJ, Otte A, Griesing T, Chambers R, Murphy TK. Pregabalin in central neuropathic pain associated with spinal cord injury: a placebo-controlled trial. Neurology. 2006;67:1792-800.

48. Cardenas DD, Warms CA, Turner JA, Marshall H, Brooke MM, Loeser JD. Efficacy of amitriptyline for relief of pain in spinal cord injury: results of a randomized controlled trial. Pain. 2002;96:365-73.

49. Mehta S, McIntyre A, Janzen S, Loh E, Teasell R. Systematic review of pharmacologic treatments of pain after spinal cord injury: an update. Arch Phys Med Rehabil. 2016;97: 1381-91.

50. Andersson DA, Gentry C, Alenmyr L, Killander D, Lewis SE, Andersson A, et al. TRPA1 mediates spinal antinociception induced by acetaminophen and the cannabinoid $\Delta$ (9)-tetrahydrocannabiorcol. Nat Commun. 2011;2:551.

51. Koivisto A, Hukkanen M, Saarnilehto M, Chapman H, Kuokkanen K, Wei H, et al. Inhibiting TRPA1 ion channel reduces loss of cutaneous nerve fiber function in diabetic animals: sustained activation of the TRPA1 channel contributes to the pathogenesis of peripheral diabetic neuropathy.Pharmacol Res. 2012;65:149-158.

52. Moran MM, McAlexander MA, Bíró T, Szallasi A. Transient receptor potential channels as therapeutic targets. Nat Rev Drug Discov. 2011;10:601-20.

53. Sałat K, Filipek BJ. Antinociceptive activity of transient receptor potential channel TRPV1, TRPA1, and TRPM8 antagonists in neurogenic and neuropathic pain models in mice. Zhejiang Univ Sci B. $2015 ; 16: 167-78$. 